\title{
On Border Traps in Back-Side-Illuminated CMOS Image Sensor Oxides
}

\author{
Andrea Vici ${ }^{\circledR}$, Felice Russo, Nicola Lovisi, and Fernanda Irrera ${ }^{\circledR}$
}

\begin{abstract}
CMOS image sensors (CISs) in back-sideilluminated configuration consist of photodiode arrays having metal lines and drive electronics beneath the active region with respect to the device/air interface so that the light reaches the photodiode active region directly. This enhances sensor quantum efficiency but reduces the electrical performance and reliability. The back-side configuration is realized by flipping the wafer upside down, bonding it to a handling wafer, mechanically thinning it, and opening a through-silicon via with a long plasma etch. As a result, gate oxides in back-side CISs show an increased density of donor-like border traps with respect to the conventional front-side-illuminated sensors. In this article, we try to add some information toward the comprehension of the origin and the electrical nature of those traps in backside gate oxides. To this aim, we performed negative bias temperature instability stress on p-channel MOSFETs during which the traps were filled by holes tunneling from the substrate and then studied the relaxation transients of the drain current after the stress was removed. The characteristic emission times of a few specific levels were obtained at different temperatures. This allowed us to extract values of the trap activation energies, which resulted coherent with hole-capturing $E^{\prime}$ donor-like centers (trivalent silicon dangling bonds) commonly attributed to ionizing radiation.
\end{abstract}

Index Terms-Back-side illuminated (BSI) CMOS image sensors (CISs), gate oxide border traps, TDDS.

\section{INTRODUCTION}

A CMOS image sensor (CIS) is an array of light-sensitive pixels. Each pixel consists of a photo-diode (PD) and several control MOSFETs. In the front-side-illuminated (FSI) configuration [Fig. 1(a)], the light reaches the PD active region through the microlenses, the filters, the passivation layers, the metal lines, and the interdielectric layers [1], [2]. So, the

Manuscript received November 26, 2019; revised February 4, 2020 and March 2, 2020; accepted March 21, 2020. The review of this article was arranged by Editor L. Pancheri. (Corresponding author: Andrea Vici.)

Andrea Vici is with the Department of Information Engineering, Electronics and Telecommunications, Sapienza University of Rome, 00185 Rome, Italy, and also with KU Leuven \& IMEC, 3001 Leuven, Belgium (e-mail: andrea.vici@imec.be).

Felice Russo and Nicola Lovisi are with LFoundry, 67051 Avezzano, Italy (e-mail: felice.russo@ Ifoundry.com; nicola.lovisi@ Ifoundry.com).

Fernanda Irrera is with the Department of Information Engineering, Electronics and Telecommunications, Sapienza University of Rome, 00185 Rome, Italy (e-mail: fernanda.irrera@ uniroma1.it).

Color versions of one or more of the figures in this article are available online at http://ieeexplore.ieee.org.

Digital Object Identifier 10.1109/TED.2020.2983039

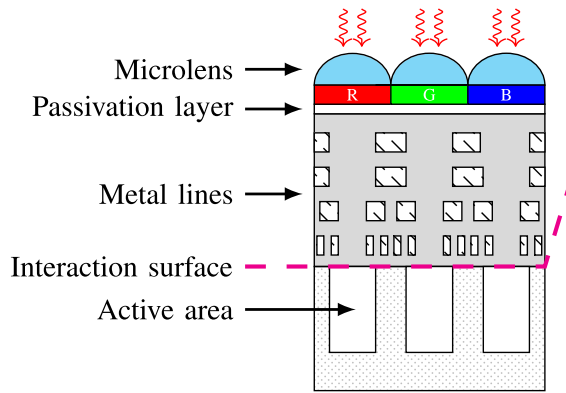

a)

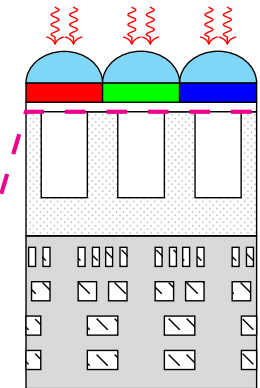

b)
Fig. 1. Cross sections of (a) front-side and (b) BSI CISs.

active region containing the PDs typically lies a few micrometers below the device/air interface. Unfortunately, the coupling of light with the front layers exhibits severe drawbacks, which cause a reduction of the maximum available photons and then a quantum efficiency degradation, especially in the blue and UV regions [3]-[6].

A few decades ago, to enhance the sensor performances, the back-side-illuminated (BSI) configuration was proposed [7]-[10]. However, due to its prohibitive production cost, the BSI configuration was set aside from the mass production for a long time, however today it is becoming popular with pixel size shrinking. The BSI configuration consists of a device having metal wiring and transistors beneath the active region with respect to the device/air interface [Fig. 1(b)] [1], [8], [11] so that the light reaches the PD active region directly, reducing loss mechanisms and crosstalk. This is achieved adding several steps with respect to FSI manufacturing process. In particular, after the deposition of metal layers, the realization of pads with oxide-nitride-oxide (ONO) passivation, and the first ${ }^{2} \mathrm{H}$ lowtemperature annealing, the BSI wafers go through a sequence consisting of flipping upside down, bonding to a handling wafer, mechanical thinning, and a long plasma etch for the through-silicon-vias (TSVs) opening. TSV opening is one of the proven technique to realize the 3-D vertical electrical connection of metal pads [12]. This sequence is here called "BSI loop." After the BSI loop, deposition of pads, ONO passivation, and a final low-temperature ${ }^{2} \mathrm{H}$ annealing are performed.

Unfortunately, despite the optical improvement, the BSI configuration exhibits several drawbacks from electrical and reliability points of view [1], [13], [14]. Some studies have been carried out to understand the reason for this 


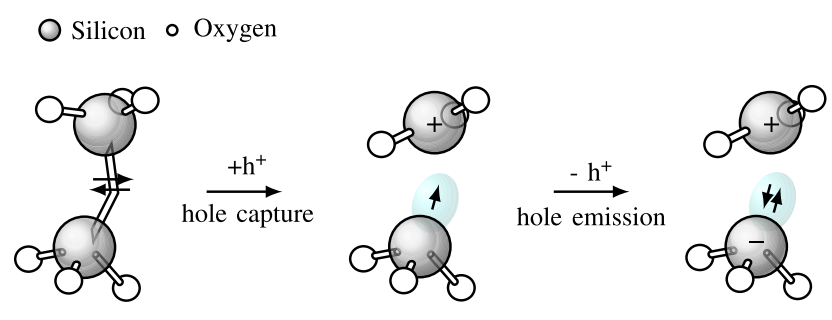

Fig. 2. Schematic representation of the switching mechanism of an $E^{\prime}$ center in $\mathrm{SiO}_{2}$, when its interaction with channel holes is probable.

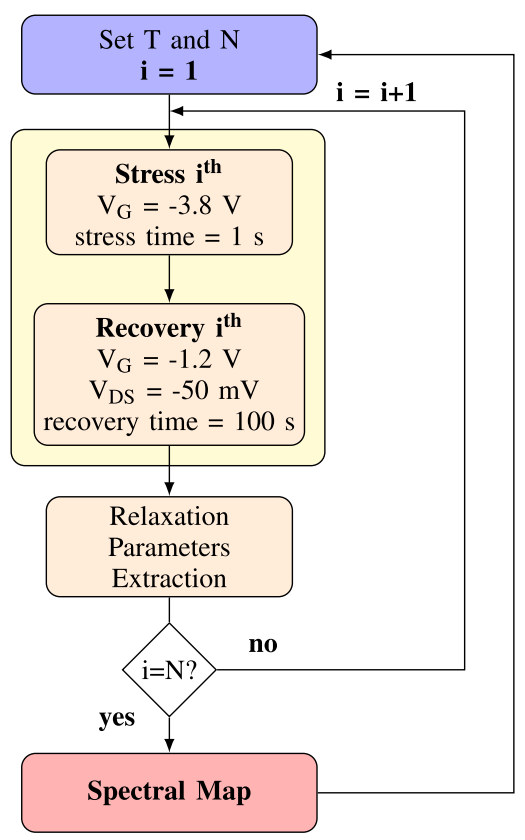

Fig. 3. Process flow of the S/R loop used in the TDDS experiment.

degradation [15]-[17], but the debate is still going on and further research must be done. In recent studies, we demonstrated that, with respect to FSI, BSI-CIS gate oxides contain an additional distribution of donor-like traps [18], [19]. These traps were located within a tunneling distance from the interface (border/slow traps), reaching a density around $10^{17} \mathrm{~cm}^{-3}$ at $1.8 \mathrm{~nm}$.

\section{ON THE ORIGIN OF BORDER TRAPS IN BSI}

The nature of traps in amorphous $\mathrm{SiO}_{2}$ is still not definitely established, however the family of defects linked to an oxygen vacancy is probably the most widely studied. Microscopically, border states are associated with $E^{\prime}$ centers (trivalent silicon dangling bonds) [20]-[22] which are defects originated on asymmetrically relaxed oxygen vacancies after hole trapping [23], [24]. A schematic representation of the mechanism by which an $E^{\prime}$ center behaves is depicted in Fig. 2. The precursors of this family of defects are two tetrahedra whose bonding oxygen is missing (left side). This structure can be modified if a hole is captured: one of the silicon atoms holds an unpaired electron on an $\mathrm{sp}^{3}$ dangling orbital and maintains a tetrahedral configuration, whereas the other holds the trapped hole and relaxes in the plane of its three-remaining oxygen neighbors (in the middle). In this situation, the $E^{\prime}$ center is positively

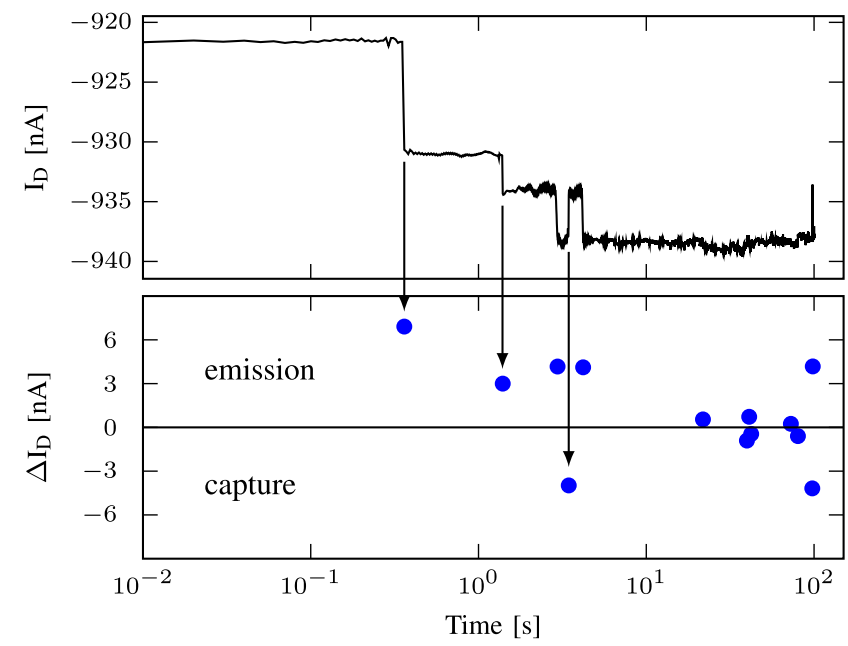

Fig. 4. Typical $I_{D}$ relaxation trace after the end of an NBTI stress and corresponding $\Delta I_{D}$ versus recovery time plot.

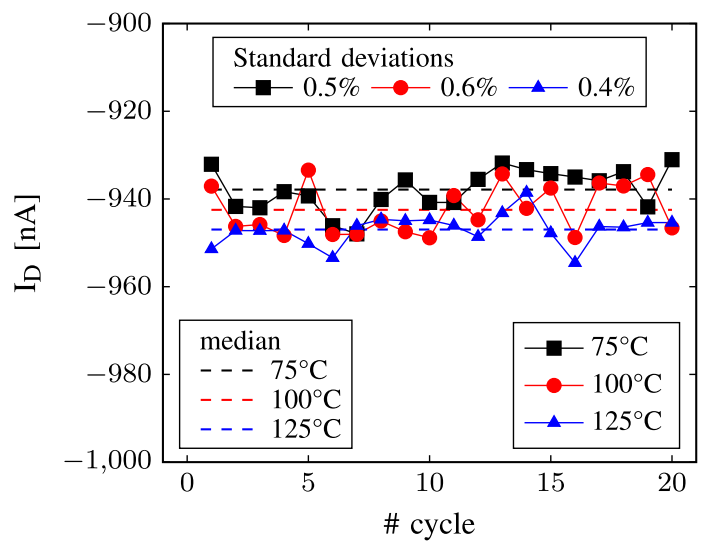

Fig. 5. $I_{D}$ measured on pMOSFET $\left(A=0.045 \mu \mathrm{m}^{2}, t_{\mathrm{ox}}=6.8 \mathrm{~nm}\right)$ during $\mathrm{S} / \mathrm{R}$ cycling for three temperatures at $V_{\mathrm{GS}}=-1.2 \mathrm{~V}$ and $V_{\mathrm{DS}}=-50 \mathrm{mV}$.

charged. Now, in the case of hole emission, the $E^{\prime}$ center switches into its neutral state (right side). So, the $E^{\prime}$ defect behaves as a donor-like trap. The fact of being positioned close to the $\mathrm{Si} / \mathrm{SiO}_{2}$ interface makes its electrical switch more probable, being the interaction with channel carriers favored by the minimal distance. The origin of this kind of donor traps is commonly attributed to ionizing radiation [25]-[31]. Unfortunately, radiation is quite common in CMOS fabrication flows especially during plasma processes, such as reactive ion etching (RIE), ashing, or plasma-enhanced chemical vapor deposition (PECVD). In our previous articles, we reported that the border traps present in BSI oxides were not found in FSI [18], [19]. This indicates that they are created during those process steps of the BSI manufacturing which are not common to the FSI configuration. As we already mentioned, in the BSI loop, there is a long and aggressive step of plasma etch for the TSV opening, and therefore, we could attribute the creation of donor-like border traps to it. Once the process step responsible for the plasma damage is identified, strategies could be proposed to reduce its effects, by, for example, acting on the recipe parameters (plasma uniformity, wafer, and plasma temperature, etch time) or using circuit solutions (protection diodes and antenna design rules) [32]-[34]. However, this will 

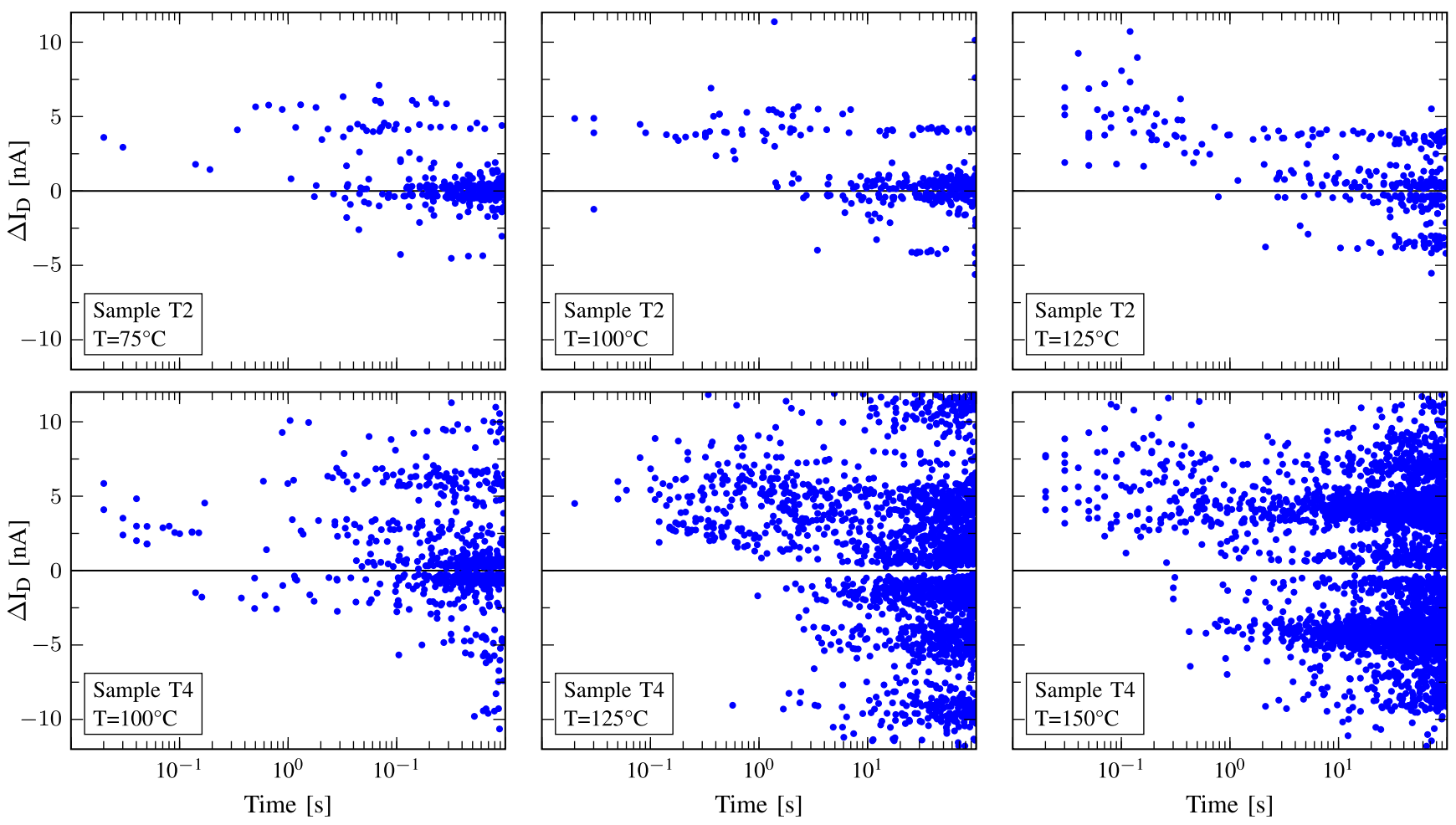

Fig. 6. Spectral maps in a $100 \mathrm{~s}$ window of the current steps at $T=75^{\circ} \mathrm{C}, 100^{\circ} \mathrm{C}, 125^{\circ} \mathrm{C}$, for samples T2 and T4 after $N$ SR cycles, where $N=25$ for T2 and $N=40$ for T4.

be demonstrated only through a systematic set of experiments before and after the TSV opening in BSI wafers, which is beyond the target of this article.

\section{Time-Dependent Defect Spectroscopy}

In this section, we add some information toward the comprehension of the electrical nature of traps created in the BSI manufacturing process, measuring their emission times and activation energies. To this aim, we show the results of a systematic study of time-dependent defect spectroscopy (TDDS) [22]-[24], [32] after negative bias temperature instability (NBTI) stresses performed at different temperatures. NBTI implies stressing p-channel MOSFETs with negative gate voltages at high temperature. This gives rise to absolute sc on-current and transconductance decrease and to absolute threshold voltage $\left(V_{T}\right)$ and subthreshold conduction increase.

A peculiar characteristic of the NBTI is its (partial) recoverability on a sufficiently long time-scale after the stress is removed [23], [24], [35].

Constant gate voltages were applied for $1 \mathrm{~s}$ and then the relaxation transients of the drain current $\left(I_{D}\right)$ were monitored for $100 \mathrm{~s}$. In large-area transistors, the $I_{D}$ relaxation transient is typically referred to follow a sort of logarithmic behavior limited to the experimental time window, although it is not mathematically correct in absolute [24]. This behavior can be modeled as the envelope of the discharge traces of many defects with widely distributed time scales. On the contrary, in small-area transistors, the recovery proceeds with discrete steps occurring at stochastic times due to the discharging of a few oxide traps. The characteristic relaxation times and step heights depend on the trap features [22], [24], [36], [37].

The analysis in this article was carried out on four pMOS transistors coming from a single production quality BSI wafer in 100-nm process technology. Four different sites were chosen in order to monitor within-wafer variability. The selected devices were wafer-level reliability (WLR) test structure, all showing $W=250 \mathrm{~nm}, L=180 \mathrm{~nm}$, and $6.8 \mathrm{~nm}$ thermally grown $\mathrm{SiO}_{2}$ oxide. The 3-D vertical electrical connection of metal pads was realized by using TSV technology. The devices were expected to be small enough to observe the step-like relaxation. Measurements were performed by using a Keithley 2612B Low Current SourceMeter, with a current resolution of $100 \mathrm{fA}$.

We obtained TDDS of each sample performing several stress/recovery (SR) cycles at different temperatures in the range $75{ }^{\circ} \mathrm{C}-150{ }^{\circ} \mathrm{C}$, in order to have a good statistics of the stochastic emission/capture processes. The SR loop is described in Fig. 3. First, the temperature and the number of cycles $(N)$ were set. Then, the $i=1$ SR cycle started. The experimental conditions for stress and recovery are indicated in Fig. 3. At the end of each cycle, the relaxation parameters (emission time $\tau_{e}$ and the current step $\Delta I_{D}=$ $\left.I_{D, \text { in }}-I_{D \text {,fin }}\right)$ were extracted. Once the $N$ cycles at the set temperature were completed, we could graph the cumulative plots of the step height versus time (the so-called spectral map [22], [35]). Then, the temperature was changed and the SR loop repeated. During the stress, the substrate is in strong inversion $\left(V_{G}=-3.8 \mathrm{~V}\right)$ and the holes capture from the substrate into the border traps is favored. When the stress is 

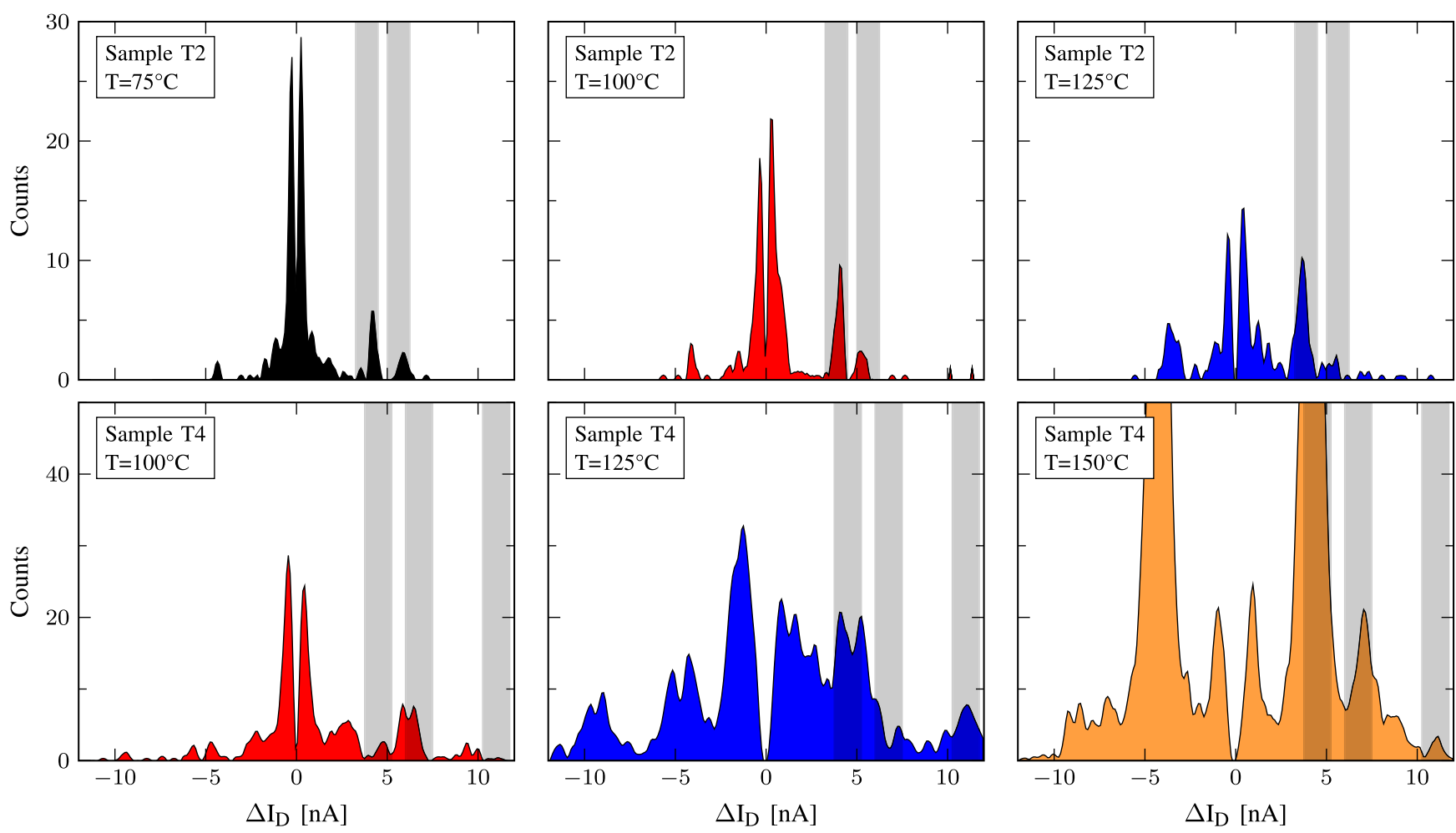

Fig. 7. $\Delta I_{D}$ distributions at different temperatures for samples T2 and T4 after $N$ SR cycles, where $N=25$ for T2 and $N=40$ for T4. The preferred $\Delta I_{D}$ levels are outlined with shadowed intervals.

removed, border traps emit holes and the $I_{D}$ current flowing when the MOSFET is slightly switched on $\left(V_{G}=-1.2 \mathrm{~V}\right)$ is influenced. In the top of Fig. 4 , a typical $I_{D}$ relaxation trace recorded after the $i$ th $\mathrm{SR}$ cycle is displayed. The $\Delta I_{D}$ current steps can be clearly observed. In this specific example, after a 400-ms long interval during which $I_{D}$ keeps constant, several steps down and very few steps up take place. A step down $\left(\Delta I_{D}>0\right)$ corresponds to the emission of a hole from a trap, while a step up $\left(\Delta I_{D}<0\right)$ corresponds to a capture. In the bottom plot of Fig. 4 , the $\Delta I_{D}$ and the relative emission/capture time $\left(\tau_{e} / \tau_{c}\right)$ corresponding to each current step are displayed. $\Delta I_{D}$ values are representative of the traps involved in the emission/capture processes, since they depend on their position in the channel potential fluctuation induced by randomly located dopants [22]-[24], [35], [36], [38]. So, the fact that more than a unique $\Delta I_{D}$ value is present indicates that more than a unique level is involved. We verified that the 1 -s long stress did not damage permanently the oxide. To this aim, we evaluated the $I_{D}$ measured at the end of each SR cycle as a function of the cycling. Results for a single sample are shown in Fig. 5, all samples behaving similarly. As one can see, the three curves are essentially constant, slightly deviating from their median values. Standard deviations are reported inside the plot. Thus, no trap creation occurs during the stress but only trap filling. Fig. 6 shows the spectral maps of two representative samples named $\mathrm{T} 2$ and $\mathrm{T} 4$, at three different temperatures. Sample T4 showed a particularly pronounced sensitivity to temperature and for this reason we report the maps up to $150^{\circ} \mathrm{C}$. Apart from fluctuations around 0 , several $\Delta I_{D}$ peaks corresponding to capture and emission processes

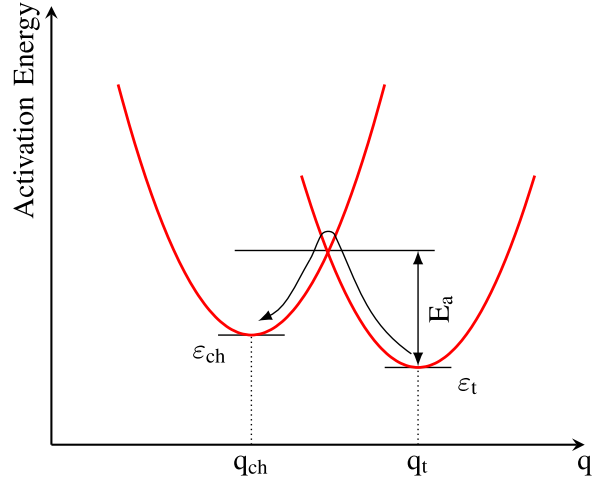

Fig. 8. Configuration coordinate diagram for hole emission from state $q_{t}$ to $q_{\mathrm{ch}}$ (after [39]).

can be clearly recognized. Other typical features are that at shorter times (up to $1 \mathrm{~s}$ approximately), many more emission events than capture occur and that the tendency to react with the same level is kept in time. The effect of temperature is better outlined in the $\Delta I_{D}$ distributions at the end of the SR cycling, displayed in Fig. 7: increasing temperature, the overall number of emission, and capture events increases and the first is always higher. In the same figure, the preferred $\Delta I_{D}$ levels are outlined with shadowed intervals, for each sample and temperature. They keep approximately constant with temperature.

Studying the behavior of $\tau_{e}$ of a trap with temperature, it is possible to extract its activation energy $\left(E_{a}\right)$. The activation energy for emission can be defined as the energy barrier that the trapped carrier must overcome to be emitted. Following the 

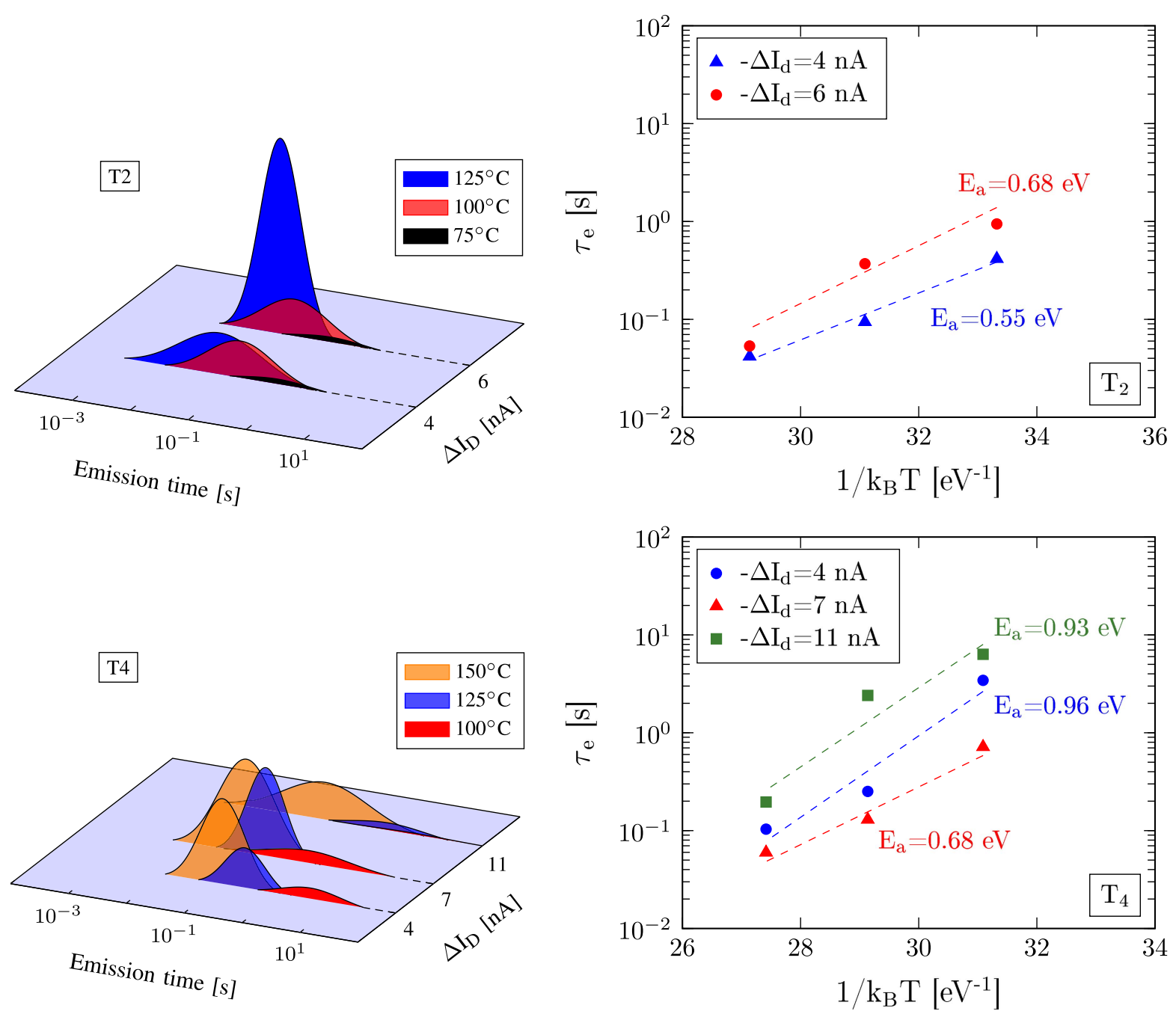

Fig. 9. Left: log-normal distributions of $\Delta I_{D}$ at different temperatures, for example, T2 and T4. Right: extraction of the activation energy of the traps involved in the SR experiment for the same samples, obtained using (1).

literature models based on nonradiative multiphonon (NMP) model [23], [24], [39], $E_{a}$ can be explained using the configuration coordinate diagram depicted in Fig. 8, where the two parabolas represent the state coordinate of the valence band in the channel $\left(q_{\mathrm{ch}}\right)$ and of a defect in the gate oxide $\left(q_{t}\right)$. In order to get a transition from state $q_{t}$ (with energy $\varepsilon_{t}$ ) to state $q_{\mathrm{ch}}$ (with an energy $\varepsilon_{\mathrm{ch}}$ ), the trapped hole must overcome the energy barrier $E_{a}$. The average emission time $\left\langle\tau_{e}\right\rangle$ is the inverse of the transition rate $k_{t \rightarrow \text { ch }}$ from state $q_{t}$ toward $q_{\text {ch }}$ and can be written as

$$
k_{t \rightarrow \mathrm{ch}}=p v_{\mathrm{th}} \sigma \cdot e^{-E_{a} / k_{\mathrm{BT}}}=\frac{1}{\left\langle\tau_{e}\right\rangle}
$$

where $p$ is the hole density, $v_{\text {th }}$ the electron thermal velocity, and $\sigma$ the trap cross section. Starting from our experiments, we derived $\left\langle\tau_{e}\right\rangle$ of each level as that time corresponding to the average value of the log-normal distribution of $\Delta I_{D}$, at each temperature. In order to distinguish and identify the most prominent peaks, we developed a reliable algorithm in MATLAB. For each sample, those peaks which recurred in the same range $( \pm 0.5 \mathrm{nA})$ at all the investigated temperatures were identified as preferred. The algorithm results indicated as preferred levels $\Delta I_{D}=4$ and $6 \mathrm{nA}$ for sample $\mathrm{T} 2$ and 4 , 7, $11 \mathrm{nA}$ for $\mathrm{T} 4$.

The 3-D plots of the log-normal distributions are shown on the left of Fig. 9, for samples T2 and T4. Then, the calculated emission times of each level can be drawn as a function of the temperature in Arrhenius-like plots, as shown on the right of Fig. 9. Now, using (1), it is possible to extract the activation energies of the traps involved in the SR experiment. As a result, the activation energies of the two main traps in $\mathrm{T} 2$ are $E_{a}=0.55 \mathrm{eV}$ and $E_{a}=0.68 \mathrm{eV}$, while in T4 the three traps feature $E_{a}=0.68,0.93$, and $0.96 \mathrm{eV}$. Activation energies of border traps in the range $0.5-1 \mathrm{eV}$ were already reported by other authors [36]-[38], [40] and attributed hole capturing to hydrogen bridges and $E^{\prime}$ centers. Indeed, even if numerous paramagnetic centers have been observed in $\mathrm{SiO}_{2}$, the results of several density functional theory calculations indicate these defects to be very likely candidates for hole trapping in $\mathrm{SiO}_{2}$ [37], [41].

\section{Conclusion}

Gate oxides in BSI CISs showed an increased density of donor-like border traps with respect to FSI sensors. TDDS has 
been performed on WLR dedicated test p-channel MOSFETs of a production quality BSI CIS wafer, monitoring relaxation of the drain current after NBTI stress performed at different temperatures. The step-like relaxation behavior typical of small size samples was found. Some features were common to all the devices studied. In particular, the interaction of holes with a few specific levels was clear and did not change with time and temperature, the emission events $\left(\Delta I_{D}\right.$ steps down) occurred always before capture events ( $\Delta I_{D}$ steps up), temperature increased the trap activity. The average emission times of each reacting level were calculated as a function of temperature to extract the corresponding activation energy, according to nonradiative multiphonon model. As a result, the activation energies of the reacting traps lay in the range $0.5-1 \mathrm{eV}$, coherently with hole capturing $E^{\prime}$ donor-like centers (trivalent silicon dangling bonds in the oxide). The origin of this kind of centers is commonly attributed to ionizing radiation during plasma processes of CMOS fabrication. We can, therefore, conclude that those donor-like BSI border traps are probably $E^{\prime}$ centers created during the etch step for the TSV opening.

\section{REFERENCES}

[1] N. Teranishi, H. Watanabe, T. Ueda, and N. Sengoku, "Evolution of optical structure in image sensors," in IEDM Tech. Dig., Dec. 2012, pp. $1-24$.

[2] R. Fontaine, "The evolution of pixel structures for consumer-grade image sensors," IEEE Trans. Semiconductor Manuf., vol. 26, no. 1, pp. 11-16, Feb. 2013.

[3] A. Lahav, A. Fenigstein, and A. Strum, "Backside illuminated (BSI) complementary metal-oxide-semiconductor (CMOS) image sensors," in High Performance Silicon Imaging. Amsterdam, The Netherlands: Elsevier, 2014, pp. 98-123.

[4] A. El Gamal and H. Eltoukhy, "CMOS image sensors," IEEE Circuits Devices Mag., vol. 21, no. 3, pp. 6-20, Jun. 2005.

[5] R. Fontaine, "Recent innovations in CMOS image sensors," in Proc. IEEE/SEMI Adv. Semiconductor Manuf. Conf., May 2011, pp. 1-5.

[6] S. Chieh, G. Agranov, H. Tian, C. Baron, H.-W. Lee, and R. Madurawe, "Challenges and opportunities in small pixel development for novel CMOS image sensors," in Proc. Int. Symp. VLSI Technol., Syst. Appl. (VLSI-TSA), Apr. 2013, pp. 1-2.

[7] R. Nixon, N. Doudoumopoulos, and E. R. Fossum, "Backside illumination of CMOS image sensor,” U.S. Patent 6429036, Aug. 6, 2002.

[8] H. Rhodes et al., "The mass production of BSI CMOS image sensors," in Proc. Int. Image Sensor Workshop, 2009, pp. 27-32.

[9] D. N. Yaung et al., "High performance $300 \mathrm{~mm}$ backside illumination technology for continuous pixel shrinkage," in IEDM Tech. Dig., Dec. 2011, pp. 2-8.

[10] E. R. Fossum and D. B. Hondongwa, "A review of the pinned photodiode for CCD and CMOS image sensors," IEEE J. Electron Devices Soc., vol. 2, no. 3, pp. 33-43, May 2014.

[11] C. Cavaco et al., "On the fabrication of backside illuminated image sensors: Bonding oxide, edge trimming and CMP rework routes," ECS Trans., vol. 64, no. 40, pp. 123-129, Apr. 2015.

[12] P. Kettner, B. Kim, S. Pargfrieder, and S. Zhu, "New technologies for advanced high density 3D packaging by using TSV process," in Proc. Int. Conf. Electron. Packag. Technol. High Density Packag., Jul. 2008, pp. $1-3$.

[13] G. Taverni et al., "Front and back illuminated dynamic and active pixel vision sensors comparison," IEEE Trans. Circuits Syst. II, Exp. Briefs, vol. 65, no. 5, pp. 677-681, May 2018.

[14] S. G. Wuu et al., "A leading-edge $0.9 \mu \mathrm{m}$ pixel CMOS image sensor technology with backside illumination: Future challenges for pixel scaling," in IEDM Tech. Dig., Dec. 2010, pp. 1-14.

[15] J. P. Gambino et al., "Device reliability for CMOS image sensors with backside through-silicon vias," in Proc. IEEE Int. Rel. Phys. Symp. (IRPS), Mar. 2018, p. 5B-6.

[16] B. Mamdy, G.-N. Lu, and F. Roy, "P-type BSI image sensor with active deep trench interface passivation for radiation-hardened imaging systems," Procedia Eng., vol. 168, pp. 176-180, 2016.
[17] Y. Sacchettini, J.-P. Carrère, V. Goiffon, and P. Magnan, "Plasma antenna charging in CMOS image sensors," in Proc. IEEE Int. Rel. Phys. Symp. (IRPS), Mar. 2019, pp. 1-5.

[18] A. Vici et al., "Through-silicon-trench in back-side-illuminated CMOS image sensors for the improvement of gate oxide long term performance," in IEDM Tech. Dig., Dec. 2018, pp. 32-33.

[19] A. Vici, F. Russo, N. Lovisi, A. Marchioni, A. Casella, and F. Irrera, "Generation of oxide traps in back-side-illuminated CMOS image sensors and impact on reliability," in Proc. 49th Eur. Solid-State Device Res. Conf. (ESSDERC), Sep. 2019, pp. 234-237.

[20] J. F. Conley, P. M. Lenahan, A. J. Lelis, and T. R. Oldham, "Electron spin resonance evidence for the structure of a switching oxide trap: Long term structural change at silicon dangling bond sites in $\mathrm{SiO}_{2}$," Appl. Phys. Lett., vol. 67, no. 15, pp. 2179-2181, Oct. 1995.

[21] P. M. Lenahan, "What can electron paramagnetic resonance tell us about the $\mathrm{Si} / \mathrm{SiO}_{2}$ system?" J. Vac. Sci. Technol. B, Microelectron., vol. 16, no. 4, pp. 2134-2153, Jul. 1998.

[22] T. Grasser, H. Reisinger, P.-J. Wagner, and B. Kaczer, “Time-dependent defect spectroscopy for characterization of border traps in metal-oxidesemiconductor transistors," Phys. Rev. B, Condens. Matter, vol. 82, no. 24, Dec. 2010, Art. no. 245318.

[23] T. Grasser et al., "The paradigm shift in understanding the bias temperature instability: From reaction-diffusion to switching oxide traps," IEEE Trans. Electron Devices, vol. 58, no. 11, pp. 3652-3666, Nov. 2011.

[24] J. Franco, B. Kaczer, and G. Groeseneken, Reliability of High Mobility SiGe Channel MOSFETs for Future CMOS Applications. Dordrecht, The Netherlands: Springer, 2014.

[25] K. P. Cheung, Plasma Charging Damage. London, U.K.: SpringerVerlag, 2001.

[26] T. P. Ma, "Plasma process induced radiation effects in CMOS technology," in Proc. 7th Int. Symp. Plasma-Process-Induced Damage, Jun. 2002, pp. 18-22.

[27] A. J. Lelis, T. R. Oldham, H. E. Boesch, and F. B. McLean, "The nature of the trapped hole annealing process," IEEE Trans. Nucl. Sci., vol. 36, no. 6, pp. 1808-1815, Dec. 1989.

[28] D. M. Fleetwood, "Reliability limiting defects in MOS gate oxides: Mechanisms and modeling implications," in Proc. IEEE Int. Rel. Phys. Symp. (IRPS), Mar. 2019, pp. 1-10.

[29] D. M. Fleetwood, "Border traps and bias-temperature instabilities in MOS devices," Microelectron. Rel., vol. 80, pp. 266-277, Jan. 2018.

[30] J. R. Schwank et al., "Radiation effects in MOS oxides," IEEE Trans. Nucl. Sci., vol. 55, no. 4, pp. 1833-1853, Aug. 2008.

[31] V. Ishchuk, B. E. Volland, M. Hauguth, M. Cooke, and I. W. Rangelow, "Charging effect simulation model used in simulations of plasma etching of silicon," J. Appl. Phys., vol. 112, no. 8, Oct. 2012, Art. no. 084308.

[32] Y. Li et al., "TSV process-induced MOS reliability degradation," in Proc. IEEE Int. Rel. Phys. Symp. (IRPS), Mar. 2018, p. 5B-5.

[33] H.-C. Shin and C. Hu, "Thin gate oxide damage due to plasma processing," Semicond. Sci. Technol., vol. 11, no. 4, p. 463, 1996.

[34] H. Shin, C.-C. King, T. Horiuchi, and C. Hu, "Thin oxide charging current during plasma etching of aluminum," IEEE Electron Device Lett., vol. 12, no. 8, pp. 404-406, Aug. 1991.

[35] T. Grasser, H. Reisinger, P.-J. Wagner, F. Schanovsky, W. Goes, and B. Kaczer, "The time dependent defect spectroscopy (TDDS) for the characterization of the bias temperature instability," in Proc. IEEE Int. Rel. Phys. Symp., May 2010, pp. 16-25.

[36] B. Kaczer et al., "A brief overview of gate oxide defect properties and their relation to MOSFET instabilities and device and circuit time-dependent variability," Microelectron. Rel., vol. 81, pp. 186-194, Feb. 2018.

[37] T. Grasser et al., "On the microscopic structure of hole traps in pMOSFETs," in IEDM Tech. Dig., Dec. 2014, pp. 1-21.

[38] T. Grasser et al., "Analytic modeling of the bias temperature instability using capture/emission time maps," in IEDM Tech. Dig., Dec. 2011, pp. 4-27.

[39] T. Grasser, "Stochastic charge trapping in oxides: From random telegraph noise to bias temperature instabilities," Microelectron. Rel., vol. 52, no. 1, pp. 39-70, Jan. 2012.

[40] W. Goes, M. Waltl, Y. Wimmer, G. Rzepa, and T. Grasser, "Advanced modeling of charge trapping: RTN, 1/f noise, SILC, and BTI," in Proc. Int. Conf. Simulation Semiconductor Processes Devices (SISPAD), Sep. 2014, pp. 77-80.

[41] D. M. Fleetwood et al., "Unified model of hole trapping, 1/f noise, and thermally stimulated current in MOS devices," IEEE Trans. Nucl. Sci., vol. 49, no. 6, pp. 2674-2683, Dec. 2002. 